Rev. Adm. Saúde Vol. 17, Nº6, Jan. - Mar. 2017

ARTIGO DE REVISÃO

\title{
Glosas Hospitalares e o Uso de Protocolos Assistenciais: Revisão Integrativa da Literatura
}

Nursing Audits and the Use of Care Protocols: Integrative Review of the Literature

\author{
Joana Angélica Santos Veloso Silva ${ }^{1}$, Sylvia Lemos Hinrichsen ${ }^{2}$, Kaline \\ Assis Carneiro Brayner ${ }^{3}$, Tatiana de Aguiar Santos Vilella ${ }^{4}$, Marcela \\ Coelho Lemos ${ }^{5}$
}

\begin{abstract}
1. Enfermeira; Hospital Memorial São José, Serviço de Auditoria; Universidade de Pernambuco, Faculdade de Ciências da Administração de Pernambuco, MBA em Gestão Hospitalar e Serviços de Saúde - Recife (PE), Brasil.
\end{abstract}

2. Médica Infectologista; Coordenadora do Núcleo de Ensino, Pesquisa e Assistência em Infectologia da UFPE; Professora Titular do Departamento de Medicina Tropical da UFPERecife (PE), Brasil.

3. Enfermeira; Hospital Memorial São José, Programa de Controle de Infecções e Gestão de Riscos/ Núcleo de Segurança do Paciente; Universidade de Pernambuco, Faculdade de Ciências da Administração de Pernambuco, MBA em Gestão Hospitalar e Serviços de SaúdeRecife (PE), Brasil.

4. Farmacêutica; Biomédica; Assistente de pesquisas do Núcleo de Ensino, Pesquisa e Assistência em Infectologia da UFPE- Recife (PE), Brasil.

5. Aluna do curso de graduação de Biomedicina da UFPE; Assistente de pesquisas do Núcleo de Ensino, Pesquisa e Assistência em Infectologia da UFPE- Recife (PE), Brasil.

\section{RESUMO}

Os registros realizados no prontuário do paciente são alvo de auditorias nas instituições de saúde devido à sua importância no cuidado ao paciente e na relação com o faturamento dos custos hospitalares. Esta revisão integrativa objetivou analisar a produção científica nacional publicada entre 2000 e 2015, sobre auditoria em enfermagem e o uso de protocolos assistenciais como ferramenta de minimização de glosas. Na busca bibliográfica, foram utilizadas as fontes de informações: BVS, SciELO, LILACS, BDENF, CAPES e Google 
Acadêmico, a partir dos descritores glosa hospitalar, protocolos assistenciais, auditoria e prontuário. A amostra constitui-se de onze trabalhos. Não foram encontrados estudos utilizando protocolos assistenciais como base de tomada de decisões no cuidado e consequentemente como elemento minimizador de glosas hospitalares. Percebe-se a necessidade de melhorar os registros do prontuário do paciente, assim como a utilização de protocolos sistematizados para controlar melhor as práticas assistenciais e os impactos na segurança do paciente.

Palavras-chave: Registros hospitalares. Protocolos. Auditoria de enfermagem. Custos hospitalares. Revisão.

\section{ABSTRACT}

The patient's medical records are subject to audit in health institutions due of its importance in patient care, as well as in relation to the billing of hospital costs. This integrative review aimed to analyze the scientific production published between 2000 and 2015 about the audit in nursing and the use of care protocols as glosses minimization tools. Bibliographic search, the sources were used information: BVS, SciELO, LILACS, BDENF, CAPES and Google Scholar, from the hospital disallowance descriptors, care protocols, auditing and records. The sample consisted of eleven papers. Studies using care protocols based decision-making in the care and consequently as minimizer element of hospital glosses were not found. There is a need to improve the records of patient quality, and the use of systematic clinical protocols with objectives to better control the care practices and their impact on patient safety.

Keywords: Hospital records. Protocols. Nursing audit. Hospital costs. Review.

\section{INTRODUÇÃO}

A atividade de auditoria tem surgido como uma ferramenta importante para mensuração da qualidade (auditoria de cuidados) e custos (auditoria de custos) das instituições de saúde ${ }^{1}$. O surgimento da auditoria está baseado na necessidade de confirmação, por parte dos investidores e proprietários, da realidade econômica e financeira espelhada no patrimônio das empresas em que investem e, principalmente em virtude do aparecimento de grandes empresas multigeograficamente distribuídas e simultâneo ao desenvolvimento econômico que propiciou a participação na formação do capital de muitas empresas ${ }^{1-18}$.

A auditoria pode ser desenvolvida em vários setores da saúde e por diferentes profissionais, destacando-se a auditoria médica e auditoria de enfermagem, 
ambas, dispondo de áreas específicas de atuação, com objetivos comuns que são o de garantir a qualidade no atendimento ao cliente, evitando desperdícios e auxiliando no controle dos custos ${ }^{1-24}$.

Um dos instrumentos imprescindíveis à auditoria é o prontuário do paciente, definido como um documento que contém informações diárias, de âmbito multiprofissional, relacionadas ao ciclo do cuidado prestado durante o processo de internação $0^{4-7}$. E, a anotação de enfermagem é um dos principais instrumentos de apoio para a análise das contas hospitalares na auditoria, que devem "registrar as condições do paciente" e sua atividade; transmitir informações acerca do paciente; prover uma base de integração e continuidade do plano total de cuidados; mostrar que as prescrições médicas foram cumpridas em relação à medicação e ao tratamento ${ }^{4,5}$.

Observa-se que as anotações efetuadas pela enfermagem consistem, portanto, no mais importante instrumento de prova de qualidade da sua atuação, uma vez que $50 \%$ das informações inerentes ao cuidado do cliente/paciente são fornecidas por esta categoria profissional, tornando indiscutível a necessidade de anotações (registros) adequados e frequentes nos prontuários do pacientes, onde devem ser relatados o estado geral do paciente, bem como os cuidados e as intercorrências que venham acontecer no período no seu cuidado ${ }^{15}$.

A glosa é considerada como o cancelamento parcial ou total do orçamento apresentado pela instituição após a prestação de seus serviços, mas, considerado pela fonte pagadora, como ilegal ou indevido, aplicada quando qualquer situação gera dúvida em relação à regra e à prática adotada pela instituição auditada $6,11,12,16$.

Boa parte das glosas hospitalares é justificada por ausência de anotações, principalmente ações das equipes de enfermagem e médica. Mas, se houvesse ações sistematizadas pelas equipes multidisciplinares, estas poderiam ser melhores delineadas através do uso de protocolos assistenciais, que facilitariam a mensuração das ações sendo, portanto, um instrumento para a auditoria de contas hospitalar, que também evitariam glosas ${ }^{6,11,12,16}$.

As ausências de informações nas anotações efetuadas no prontuário são frequentes quando se tem problemas de compreensão da letra, com isso o índice de glosas tem sido significativo, aumentando assim as glosas efetuadas, levando a um valor considerável à instituição ${ }^{7-9,14}$.

Os registros do prontuário do cliente/paciente são também utilizados para fins de faturamento/cobrança, para auditoria interna ou externa, para obtenção de dados estatísticos sobre as atividades realizadas e para análise institucional. É de interesse de todos os envolvidos nos processos de análises de faturamento, tanto do hospital como do plano de saúde, que sejam anotadas, com qualidades e exatidão, as informações no prontuário, pois através delas serão avaliados os procedimentos realizados entre outros itens, como materiais e medicamentos, o que repercutirá na estabilidade financeira das instituições. Assim, é indispensável neste processo o pleno conhecimento da equipe, a padronização do processo de trabalho com protocolos, a capacitação contínua 
e sistematizada, a reflexão sobre o conteúdo das informações e dos impressos para anotação ${ }^{6-9,14}$.

Nos últimos anos, tem sido observada uma maior preocupação no processo de qualidade e assistência ao paciente, focado em padrões de qualidade e acreditação, que utilizam ferramentas de gestão no ciclo do cuidado que garantem maior conformidade nos registros de prontuários do paciente utilizando evidências científicas e protocolos assistenciais como norteadores de qualidade institucional e da melhoria da auditoria e de glosas ${ }^{5}$.

Define-se como protocolos assistenciais, orientações sistematizadas, às vezes em formato de fluxograma ou de uma matriz temporal, baseados nas diretrizes e evidências da literatura e elaborados por especialistas de uma instituição na qual serão implementados. Priorizam pontos críticos e básicos no processo de decisão. É uma atividade que agrega valor ao negócio, mas que precisa de pessoas envolvidas, responsáveis, comunicando-se. Não é apenas um preenchimento de ficha, mas, um retrato do que precisa e precisou ser feito no ciclo do cuidado do paciente. Através de uma assistência sistematizada utilizando protocolos assistenciais poder-se-á conseguir:1- um equilíbrio entre os requisitos dos clientes e a melhoria da qualidade; 2-prover treinamentos apropriados; 3-assegurar a rastreabilidade e repetitividade; 4-prover evidências objetivas; 5-avaliar a eficácia e a contínua adequação do sistema de gestão. A padronização dos protocolos assistenciais coleta, organiza, analisa e descreve idéias, assim como verifica e padroniza, servindo de base para indicadores de riscos/qualidade.

Os protocolos devem cumprir três funções: 1- gerencial (controlar a lei da variabilidade clínica nos serviços de saúde, instrumentalizar os profissionais na tomada de decisão, homogeneizar as condutas clínicas); 2- educacional (produto de treinamentos e educação), e 3- comunicação (educar os profissionais e o paciente/usuário em relação às condições da doença e saúde $)^{5}$.

Com base no exposto, o presente trabalho foi proposto com o objetivo de evidenciar qual a produção científica acerca da importância do uso de protocolos assistenciais na diminuição de glosas hospitalares, através da auditoria, tendo como base uma revisão sobre o tema na literatura.

\section{METODOLOGIA}

Trata-se de uma revisão integrativa da literatura referente à produção científica a respeito do tema em questão. Esse método viabiliza a análise de pesquisas científicas de modo sistemático e amplo e favorece a caracterização e a divulgação do conhecimento produzido ${ }^{16}$. Para a sua elaboração, a metodologia foi operacionalizada por meio das seguintes etapas:

estabelecimento da questão norteadora da pesquisa, busca na literatura, coleta de dados, análise crítica dos estudos incluídos, interpretação e síntese dos resultados e apresentação da revisão. 
A busca online foi realizada por meio da Biblioteca Virtual em Saúde (BVS), na base de dados eletrônica Scientific Electronic Library Online (SciELO), Literatura Latino-americana e do Caribe de Informação em Ciências da Saúde (LILACS) e Base de Dados de Enfermagem (BDENF); Portal da Coordenação de Aperfeiçoamento de Pessoal de Nível Superior (CAPES), e, Google Acadêmico. Para operacionalizar a busca, utilizaram-se as palavras-chaves: glosa hospitalar, protocolos assistenciais e auditoria, a partir dos descritores: registros hospitalares; protocolos; auditoria de enfermagem; custos hospitalares; revisão (DeCS- http:/decs.bvs.br).

A seleção dos artigos foi realizada no período de 2000 a 2015, por meio dos seguintes critérios de inclusão: artigos periódicos online nacionais indexados nas bases de dados, e cujos textos estivessem apresentados na íntegra, retratando a temática e publicados no período do estudo, oriundos de estudos desenvolvidos no Brasil, em português e relacionados aos descritores selecionados.

Para a seleção dos artigos realizou-se, inicialmente, a leitura dos resumos das publicações selecionadas com o objetivo de refinar a amostra por meio de critérios de inclusão e exclusão.

Os critérios de exclusão foram: artigos com ausência de resumo nas plataformas de busca on-line, artigos de reflexões e ou os que não estivessem associados aos descritores/palavras-chaves selecionados.

A avaliação crítica dos artigos constituiu, na literatura, o estudo na íntegra, em seguida na sintetização de artigos selecionados. Para tal, elaborou-se um instrumento de coleta de dados, que foi preenchido para cada artigo da amostra final, contendo as seguintes variáveis: referência (autor, data), objetivos, fonte de busca e resultados encontrados.

Após a busca, foram identificados vários artigos que não enquadraram nos critérios de inclusão. Na sintetização dos artigos selecionados, elaborou-se um instrumento de coleta de dados, preenchido para cada artigo da amostra final, contendo as seguintes variáveis: referência (autor, data), bases de dados, objetivos e resultados encontrados.

Os dados evidenciados na análise foram discutidos segundo literatura e apresentado de forma descritiva, com fins de possibilitar a aplicabilidade prática da auditoria nos registros de enfermagem, focados nas causas de glosas hospitalares e se uso de protocolos assistenciais que possam minimizar estas.

\section{RESULTADOS}

Na revisão bibliográfica realizada foram identificados vários artigos relacionados à auditoria, auditoria de enfermagem, porém 11 artigos atenderam aos critérios de inclusão previamente estabelecidos, segundo palavras-chaves e descritores selecionados que podem ser observados no Quadro 1, e que contém as variáveis estudadas, no período de 2000 a 2015. 
Dos vários artigos identificados, observaram-se 139 relacionados às palavraschaves selecionadas: glosas e prontuários; glosas hospitalares; glosas e prontuários assistenciais; protocolos assistenciais sem glosas e ou auditorias de prontuários; e protocolos assistenciais e auditorias, auditorias de enfermagem. Foram encontrados 4.094 artigos relacionados a auditoria de enfermagem e 32.906 em auditoria.

Não foram encontrados estudos que estivessem correlacionados ao processo de auditorias de prontuários e ou glosas utilizando protocolos assistenciais, baseados em evidências científicas, como ferramenta para sistematizar a coleta de registros da assistência prestada segundo plano diagnóstico e ou terapêutico, que consequentemente minimizassem as perdas de registros relacionados aos serviços prestados pela instituição ao paciente.

Em relação aos anos em que foram publicados os artigos estudados, segundo descritores utilizados, não foram encontrados estudos nos anos: 2000, 2001, 2005, 2006, 2010, 2011 e 2014. No ano de 2002 foi encontrado um artigo com os critérios de inclusão e nos demais observaram-se: $2003(n=1) ; 2004(n=1)$; $2007(n=1) ; 2008(n=3) ; 2009(n=1) ; 2012(n=1) ; 2013(n=1)$ e $2015(n=1)$. A base de dados, fonte, mais encontrada foi LILACS $(n=8)$ (Quadro 1$)$.

Todos os estudos (100\%) estiveram relacionados à importância da enfermagem e ou das equipes no processo de auditoria, assim como às principais não conformidades encontradas que levaram às glosas dos serviços prestados assim como os seus impactos e ou dificuldades advindas destas no processo de cobranças e ou perdas financeiras institucionais durante a assistência prestada (Quadros 1, 2).

Quadro 1. Dados sobre a referência, ano, fonte de dados, objetivos e resultados encontrados dos estudos selecionados.

\begin{tabular}{|l|l|l|l|}
\hline Autor & $\begin{array}{l}\text { ANO, FONTE } \\
\text { DE DADOS }\end{array}$ & OBJETIVOS & $\begin{array}{l}\text { RESULTADOS } \\
\text { ENCONTRADOS }\end{array}$ \\
\hline GALVÃO, CF & 2002, SciELO & $\begin{array}{l}\text { Investigar o papel da } \\
\text { auditoria de enfermagem } \\
\text { na redução de } \\
\text { desperdícios de materiais } \\
\text { e medicamentos, } \\
\text { considerando-a como } \\
\text { uma ferramenta } \\
\text { administrativa para o } \\
\text { controle de custos. }\end{array}$ & $\begin{array}{l}\text { Verificados os registros } \\
\text { incorretos da equipe de } \\
\text { enfermagem com relação } \\
\text { aos medicamentos e } \\
\text { materiais. Não há um } \\
\text { trabalho proativo para a } \\
\text { redução de desperdícios } \\
\text { hospitalares em materiais } \\
\text { e medicamentos, sendo } \\
\text { necessária a revisão de } \\
\text { suas rotinas, bem como a } \\
\text { implantação de um } \\
\text { treinamento e a } \\
\text { conscientização da } \\
\text { equipe de enfermagem, a } \\
\text { fim de que todos sejam } \\
\text { informados dos recursos } \\
\text { econômico-financeiros do } \\
\text { hospital, uma vez que }\end{array}$ \\
\hline & &
\end{tabular}




\begin{tabular}{|c|c|c|c|}
\hline & & & $\begin{array}{l}\text { valores não pagos pelos } \\
\text { convênios médicos, em } \\
\text { decorrência de falhas nos } \\
\text { registros de enfermagem, } \\
\text { são significativos. }\end{array}$ \\
\hline RIOLINO, NA & 2003, LILACS & $\begin{array}{l}\text { Relata a atuação de } \\
\text { enfermeiras que exercem } \\
\text { a função de Auditoras de } \\
\text { Prontuário, considerado } \\
\text { como o comprovante de } \\
\text { dados e ações realizadas } \\
\text { durante a internação e a } \\
\text { auditoria, embora } \\
\text { usualmente atuante como } \\
\text { agente controlador dos } \\
\text { custos, tem aqui } \\
\text { aplicação sobre outro } \\
\text { prisma, visando a } \\
\text { conformidade de } \\
\text { informações baseada em } \\
\text { padrões institucionais e } \\
\text { princípios éticos e legais. } \\
\text { O principal objetivo foi o } \\
\text { de promover ações de } \\
\text { melhorias a partir dos } \\
\text { resultados encontrados, } \\
\text { em conjunto com as } \\
\text { equipes multiprofissionais }\end{array}$ & $\begin{array}{l}\text { Observado uma maior } \\
\text { preocupação de toda } \\
\text { instituição quanto ao } \\
\text { correto preenchimento da } \\
\text { documentação e que o } \\
\text { enfermeiro auditor de } \\
\text { prontuário é uma } \\
\text { tendência, com campo de } \\
\text { atuação vasto e } \\
\text { significativo, garantindo } \\
\text { importante inserção e } \\
\text { reconhecimento no } \\
\text { mercado. Onde a } \\
\text { enfermagem realizará um } \\
\text { maior número de } \\
\text { anotações extensas e } \\
\text { pouco específicas, mais } \\
\text { legíveis e objetivas. }\end{array}$ \\
\hline $\begin{array}{l}\text { RODRIGUES, } \\
\vee A^{8}\end{array}$ & 2004, LILACS & $\begin{array}{l}\text { Estudo retrospectivo de } \\
\text { natureza exploratória } \\
\text { descritiva, com objetivo } \\
\text { de investigar os fatores } \\
\text { intervenientes nas glosas } \\
\text { ocorridas em um hospital } \\
\text { de ensino. A coleta de } \\
\text { dados incluiu as glosas } \\
\text { identificadas a partir de } \\
\text { seus recursos, junto a } \\
\text { duas operadoras de } \\
\text { planos de saúde, no } \\
\text { período de janeiro a } \\
\text { março de } 2003 \text {. }\end{array}$ & $\begin{array}{l}\text { Foram encontrados } \\
17.324 \text { itens glosados nos } \\
85 \text { recursos de glosas } \\
\text { analisados, os quais } \\
\text { representaram um valor } \\
\text { de } R \$ 31.856,52 \text {. Os } \\
\text { recursos de glosas para } \\
\text { materiais representaram } \\
55 \% \text { do valor total. Cerca } \\
\text { de } 99,6 \% \text { dos recursos de } \\
\text { glosas para materiais } \\
\text { foram embasados nas } \\
\text { "anotações e/ou } \\
\text { checagem de } \\
\text { enfermagem". } \\
\text { Correlacionando o valor } \\
\text { dos recursos de glosas } \\
\text { com as justificativas } \\
\text { relacionadas com a } \\
\text { equipe de enfermagem } \\
\text { notou-se que essas } \\
\text { últimas justificavam o } \\
\text { estorno de } R \$ 22.877,80 \text {. } \\
\text { Conclui-se que a prática } \\
\text { de anotação de } \\
\text { enfermagem é de extrema } \\
\text { importância para as } \\
\text { instituições de saúde uma } \\
\text { vez que a partir dela é } \\
\text { possível reaver cerca de }\end{array}$ \\
\hline
\end{tabular}




\begin{tabular}{|c|c|c|c|}
\hline & & & $\begin{array}{l}87,7 \% \text { do custo total } \\
\text { glosado pelos de planos } \\
\text { de saúde }\end{array}$ \\
\hline LUZ, $A^{9}$ & 2007, LILACS & $\begin{array}{l}\text { Identificar a qualidade dos } \\
\text { registros de enfermagem } \\
\text { em contas hospitalares. }\end{array}$ & $\begin{array}{l}\text { Observadas anotações } \\
\text { realizadas por turno e não } \\
\text { por horário, rasuras nas } \\
\text { escritas e espaços em } \\
\text { branco ao longo do } \\
\text { impresso, falta de carimbo } \\
\text { e de assinatura. Há } \\
\text { prontuários em que a } \\
\text { checagem de prescrições } \\
\text { não ocorre ou é realizada } \\
\text { de forma incorreta; há } \\
\text { também anotação } \\
\text { incompleta de sinais } \\
\text { vitais. De um modo geral } \\
\text { as anotações são } \\
\text { compreensíveis embora a } \\
\text { letra seja pouco legível e } \\
\text { há a utilização de siglas } \\
\text { padronizadas e termos } \\
\text { técnicos. }\end{array}$ \\
\hline GODOI, AP ${ }^{10}$ & 2008, LILACS & $\begin{array}{l}\text { Avaliar os indicadores } \\
\text { mais frequentes nas } \\
\text { discussões hospitalares } \\
\text { através de uma avaliação } \\
\text { dos prontuários e das } \\
\text { anotações de } \\
\text { enfermagem, para a } \\
\text { diminuição destes índices } \\
\text { de maneira global. }\end{array}$ & $\begin{array}{l}\text { Observada inconsistência, } \\
\text { incoerência entre } \\
\text { prescrição, evolução } \\
\text { clínica, anotação e } \\
\text { checagem de } \\
\text { enfermagem. }\end{array}$ \\
\hline $\begin{array}{l}\text { SCARPARO, } \\
\text { AF }^{11}\end{array}$ & 2008, BDENF & $\begin{array}{l}\text { Identificar e analisar } \\
\text { opiniões de especialistas } \\
\text { em auditoria que atuam } \\
\text { no contexto da } \\
\text { enfermagem e } \\
\text { sistematizar tendências } \\
\text { de concepção, método e } \\
\text { finalidade da auditoria de } \\
\text { enfermagem, utilizando- } \\
\text { se a técnica Delphi, que } \\
\text { consiste em um tipo de } \\
\text { avaliação prospectiva e } \\
\text { consensual de } \\
\text { tendências, por parte de } \\
\text { especialistas no tema } \\
\text { investigado. Uma técnica } \\
\text { indicada quando há } \\
\text { inexistência de dados, } \\
\text { necessidade de } \\
\text { abordagem } \\
\text { multidisciplinar ou mesmo } \\
\text { quando há falta de } \\
\text { consenso em } \\
\text { determinado assunto. }\end{array}$ & $\begin{array}{l}\text { Os resultados revelaram } \\
\text { que a concepção da } \\
\text { auditoria está focada na } \\
\text { visão contábil e } \\
\text { financeira, tendo em vista } \\
\text { a sustentação econômica } \\
\text { do hospital e como ato de } \\
\text { controladoria visando } \\
\text { identificar pagamentos } \\
\text { indevidos referentes à } \\
\text { conta hospitalar; que no } \\
\text { futuro, a essa concepção, } \\
\text { será associada à } \\
\text { avaliação da qualidade da } \\
\text { assistência, com } \\
\text { envolvimento em outras } \\
\text { áreas que nela interferem. }\end{array}$ \\
\hline
\end{tabular}




\begin{tabular}{|c|c|c|c|}
\hline $\begin{array}{l}\text { CIANCIRULL } \\
\mathrm{O}, \mathrm{TI}^{12}\end{array}$ & 2008, SciELO & $\begin{array}{l}\text { Analisar o sistema de } \\
\text { assistência de } \\
\text { enfermagem }\end{array}$ & $\begin{array}{l}\text { Foi verificado que a } \\
\text { assistência de } \\
\text { enfermagem prestada } \\
\text { relacionada a sua } \\
\text { evolução e tendência, } \\
\text { visa um trabalho proativo } \\
\text { para a redução de } \\
\text { desperdícios hospitalares } \\
\text { em materiais e } \\
\text { medicamentos, sendo } \\
\text { necessária a revisão de } \\
\text { suas rotinas, bem como a } \\
\text { implantação de um } \\
\text { treinamento e a } \\
\text { conscientização da } \\
\text { equipe de enfermagem. }\end{array}$ \\
\hline $\begin{array}{l}\text { FERREIRA, } \\
\text { TS }^{13}\end{array}$ & 2009, LILACS & $\begin{array}{l}\text { Identificar o impacto } \\
\text { causado pelo não registro } \\
\text { de enfermagem } \\
\text { contrapondo às eventuais } \\
\text { glosas, evidenciando os } \\
\text { principais tipos } \\
\text { decorrentes destes } \\
\text { registros. }\end{array}$ & $\begin{array}{l}\text { Observado que a } \\
\text { anotação de enfermagem } \\
\text { não deve ser encarada } \\
\text { como um simples } \\
\text { cumprimento de norma } \\
\text { burocrática, passível de } \\
\text { esquecimento. É preciso } \\
\text { que se tenha a noção de } \\
\text { sua real importância e das } \\
\text { implicações decorrentes } \\
\text { do não preenchimento } \\
\text { correto deste documento. } \\
\text { A conseqüência } \\
\text { observada foram glosas } \\
\text { em medicamentos } \\
(53,0 \%) \text {; taxas e aluguéis } \\
(2,9 \%) \text { e de materiais } \\
(23,0 \%) \text {. }\end{array}$ \\
\hline $\begin{array}{l}\text { MULATINHO, } \\
\text { LM }^{14}\end{array}$ & $\begin{array}{l}\text { 2012, Google } \\
\text { Acadêmico }\end{array}$ & $\begin{array}{l}\text { Avaliar os fatores } \\
\text { determinantes das glosas } \\
\text { hospitalares }\end{array}$ & $\begin{array}{l}\text { Identificados } 13 \text { itens } \\
\text { glosados, somando um } \\
\text { total de } 215 \text { glosas, } \\
\text { dessas, } 148(68,84 \%) \\
\text { foram glosas de } \\
\text { medicamentos e } 67 \\
(31,16 \%) \text { glosas de } \\
\text { materiais. As glosas } \\
\text { referentes aos } \\
\text { medicamentos foram } \\
\text { classificadas como } \\
\text { técnicas, já as glosas dos } \\
\text { materiais como técnicas e } \\
\text { administrativas. }\end{array}$ \\
\hline $\begin{array}{l}\text { PEREIRA } \\
\text { DOS } \\
\text { SANTOS }^{15}\end{array}$ & $\begin{array}{l}\text { 2013, Google } \\
\text { Acadêmico }\end{array}$ & $\begin{array}{l}\text { Investigar os motivos de } \\
\text { glosas ocorridas em um } \\
\text { hospital da cidade de São } \\
\text { Paulo, através da coleta } \\
\text { de dados de glosas } \\
\text { identificadas a partir de } \\
\text { seus recursos, junto a }\end{array}$ & $\begin{array}{l}\text { Foram encontrados } 921 \\
\text { itens glosados nos } \\
\text { recursos analisados, cujo } \\
\text { valor total foi de } \mathrm{R} \$ \\
173.603,36 \text {. Noventa e } \\
\text { um por cento dos } \\
\text { recursos referem-se a }\end{array}$ \\
\hline
\end{tabular}




\begin{tabular}{|c|c|c|c|}
\hline & & $\begin{array}{l}\text { uma operadora de planos } \\
\text { de saúde. }\end{array}$ & $\begin{array}{l}\text { glosas administrativas e } \\
9 \% \text { à glosas técnicas. } \\
\text { Quando o valor é } \\
\text { recusado cerca de } 22 \% \\
\text { são acatados. Os } \\
\text { resultados finais } \\
\text { revelaram que } 75 \% \text { das } \\
\text { glosas ocorridas podem } \\
\text { ser evitadas. }\end{array}$ \\
\hline $\begin{array}{l}\text { GUERRER, } \\
\text { GFF }^{16}\end{array}$ & $\begin{array}{l}\text { 2015, } \\
\text { LILACS/SciELO }\end{array}$ & $\begin{array}{l}\text { Através de pesquisa } \\
\text { quantitativa exploratória, } \\
\text { descritiva do tipo estudo } \\
\text { de caso único foram } \\
\text { verificados os itens } \\
\text { componentes das contas } \\
\text { hospitalares conferidos } \\
\text { por enfermeiros auditores, } \\
\text { que mais recebem ajustes } \\
\text { no momento da pré- } \\
\text { análise, identificando o } \\
\text { impacto dos ajustes no } \\
\text { faturamento das contas } \\
\text { analisadas por } \\
\text { enfermeiros e médicos } \\
\text { auditores e as glosas } \\
\text { relacionadas aos itens } \\
\text { conferidos pela equipe de } \\
\text { auditoria. }\end{array}$ & $\begin{array}{l}\text { Após a análise de } 2.613 \\
\text { contas constatou-se que o } \\
\text { item mais incluído por } \\
\text { enfermeiros foram gases } \\
\text { (90,5\%) e o mais excluído } \\
\text { medicamentos de } \\
\text { internação ( } 41,2 \%) \text {. } \\
\text { Materiais de } \\
\text { hemodinâmica; gases e } \\
\text { equipamentos foram os } \\
\text { que mais impactaram nos } \\
\text { ajustes positivos. Os } \\
\text { ajustes negativos } \\
\text { decorreram de } \\
\text { lançamentos indevidos } \\
\text { nas contas e não geraram } \\
\text { prejuízos de faturamento. } \\
\text { Do total de glosas } 52,24 \% \\
\text { referiu-se à pré-análise } \\
\text { dos enfermeiros e } 47,76 \% \\
\text { a dos médicos. }\end{array}$ \\
\hline
\end{tabular}

Quadro 2. Principais não conformidades observadas nas anotações em prontuários segundo referência (autor data).

\begin{tabular}{|l|l|}
\hline NÃO CONFORMIDADE & AUTOR, DATA \\
\hline $\begin{array}{l}\text { Registros incorretos da equipe de enfermagem com relação } \\
\text { aos medicamentos e materiais }\end{array}$ & GALVÃO, CF $; 2002$ \\
\hline Anotações extensas e pouco específicas & RIOLINO, NA $; 2003^{7} ;$ \\
\hline $\begin{array}{l}\text { Falta de carimbo e de assinatura } \\
\text { Letra poucoão incompleta de sinais vitais }\end{array}$ & \\
Anotações realizadas por turno e não por horário & \\
Rasuras nas escritas & \\
Espaços em branco ao longo do impresso & \\
\hline
\end{tabular}




\begin{tabular}{|l|l|}
\hline $\begin{array}{l}\text { Falta de assinatura } \\
\text { Checagem de prescrições não ocorridas ou realizadas }\end{array}$ \\
\hline $\begin{array}{l}\text { Inconsistência, incoerência entre prescrição, evolução clínica, } \\
\text { anotação e checagem }\end{array}$ & GODOI, AP $^{10} ; 2008$ \\
\hline $\begin{array}{l}\text { Exclusão de medicamentos de internação; materiais de } \\
\text { hemodinâmica; gases e equipamentos. } \\
\text { Lançamentos indevidos nas contas }\end{array}$ & GUERRER,GFF $^{16} ; 015$ \\
\hline
\end{tabular}

\section{DISCUSSÃO}

O processo de auditoria é conceituado como uma avaliação sistemática e formal de uma atividade realizada por pessoas não envolvidas diretamente em sua execução, a fim de se determinar se a atividade está de acordo com os objetivos propostos sobre o tema em estudo ${ }^{1-18}$. O termo 'auditoria' refere-se a avaliação, exame analítico e pericial que segue o desenvolvimento das operações e atividades, sejam elas contábeis desde o início até o balanço ou outras $^{1,13,17}$.

$\mathrm{Na}$ área da saúde, a auditoria tem ampliado seu campo de atuação para a análise da assistência multiprofissional prestada, tendo em vista a qualidade e seus envolvidos, que são o paciente, o hospital, os colaboradores e as operadoras de saúde, conferindo os procedimentos executados com os valores cobrados para garantir um pagamento justo. Assim, a análise realizada envolve aspectos quantitativos e qualitativos da assistência, ou seja, a avaliação da eficácia e eficiência do processo de atenção à saúde onde estão incluídas todas as atividades realizadas ${ }^{5-16}$.

A auditoria hospitalar deve ter um foco preventivo, que deverá buscar uma orientação, numa linguagem única, que deve ser realizada pelo serviço de auditoria da instituição, com bases nas práticas realizadas pelos multiprofissionais que assistem ao paciente com qualidade, com economia e redução de custos. É, portanto, um processo que vem sendo estabelecido nas instituições de saúde como um todo, pois trata-se de uma ferramenta controladora de custos e de qualidade assistencial, focada, preferencialmente, em protocolos clínicos, no qual os profissionais da enfermagem e outros, tem entendido esta questão e trabalhado para contribuir que o fluxo e documentos utilizados sejam os mais adequados ${ }^{1,5-16}$.

Em relação a auditoria de custos, esta tem como finalidade conferir e controlar o faturamento das contas das atividades geradas durante a assistência prestada e que são enviadas para as fontes pagadoras, além de verificar exames e procedimentos realizados, efetuar visitas de rotina a pacientes internados, cruzando as informações recebidas com as que constam no prontuário do paciente ${ }^{6-17}$. Visa, também, investigar a propriedade dos gastos e processos de pagamentos; analisar as estatísticas, indicadores hospitalares e 
específicos da organização; conferir os sistemas de faturamento das contas médicas e, ainda, elaborar processos de glosas contratuais e administrativas ${ }^{6-}$ 17.

Discute-se muito sobre custo, mas, pouco o define para uma melhor compreensão. Assim, custo é utilização normal de bens ou serviços no processo de produção de outros bens ou serviços, devendo satisfazer a dois requisitos: 1- ocorrência de utilização de um recurso econômico, e, 2- o objetivo de produzir bens ou serviços, para ser, então considerado como custo, que exigirá o recurso de normalidade e previsibilidade dentro do processo produtivo ${ }^{1,5,25}$. Mas, se a utilização ocorrer de maneira anormal, em decorrência de um sinistro, por exemplo, a literatura contábil o classifica como perda, pois não é necessário à produção. Na prestação de serviços, a produção e o consumo ocorrem simultaneamente, pois os serviços não são estocáveis ${ }^{1,5,25}$

Nas 11 (100,0\%) publicações estudadas, o que chama atenção é a referência das dificuldades encontradas nas auditorias de enfermagem relacionadas à qualidade dos registros de prontuários que dificultam a obtenção de informações que poderão gerar inseguranças na prática assistencial do paciente, assim como déficit nas cobranças dos serviços prestados pela instituição de saúde/ hospital. Não conformidades, em registros de atividades prestadas no cuidado do paciente, sem a menor dúvida interferem na quantificação do trabalho realizado, nos custos gerados por eles, pela falta de evidências do que foi executado durante o cuidado prestado ao paciente, o que gerará glosas por parte das fontes pagadoras.

Observou-se na revisão realizada que, quanto maior o número de falta de registros dos produtos/atividades gerados consequentes à assistência prestada, maior serão as oportunidades de glosas, isto é, os não pagamentos pelo serviço realizado.

Também não se observa, nestes estudos, a necessidade de se ter um sistema uniforme do processo de auditoria que foque em atividades não só assistenciais que guiem os procedimentos realizados conforme fluxos e ou evidências científicas. Ainda são muito básicas as não conformidades encontradas nos registros e ou não registradas que dificultam o processo do auditar corretamente. Percebe-se nas atividades realizadas pelos multiprofissionais, nos estudos avaliados, a existência de falhas do processo de auditar, sem a intervenção em melhorias de forma sistemática e processual. Registram-se as não conformidades, mas, não são incluídos os planos de ações para que ocorram as mudanças necessárias, embora, haja sugestões de processos educacionais das equipes multiprofissionais como alternativa de melhorias no processo de registros em prontuários.

Observou-se, também, que as glosas ou correções são aplicadas quando qualquer situação gera dúvidas em relação às regras e às práticas adotadas pela instituição de saúde. E, quando elas acontecem, observa-se a existência de conflito na relação entre a fonte pagadora e o prestador de serviços, fazendo com que as instituições de cuidado à saúde que têm os seus valores dos serviços prestados glosados pelas fontes pagadoras, lancem mão de recursos denominados "recursos de glosas", a fim de recuperar suas perdas 
econômicas. Mas, mesmo com uma nova oportunidade de recorrer às glosas, a instituição perde tempo em receber os aportes financeiros, que foram gastos durante o ciclo do cuidado ao paciente.

Interessante observar nos estudos $(n=11)$ que o motivo das glosas e ou das dificuldades na auditoria dos serviços prestados pelas instituições está relacionado, na grande maioria, à falta de registros em prontuários do paciente dos itens utilizados durante a assistência prestada. Também a ilegibilidade dos registros em prontuários é outro fator de importância a ser considerado, e, um forte argumento para que as instituições busquem ações de melhorias que poderão estar relacionadas e justificadas à implantação de prontuários eletrônicos, que podem auxiliar neste processo de perdas de informações registradas.

Sabe-se nos dias atuais a importância de se assistir aos pacientes nas instituições de saúde/ hospitais focadas em referências na gestão do conhecimento e nas evidências científicas que são, tanto provedoras à empresa de subsídios que garantem esta efetividade, qualidade, velocidade de mudanças, ajustes e inovação, quanto ao cliente do processo, à medida que depende de inputs de estratégia para realinhá-la constantemente ${ }^{5,26,27}$.

Conceitua-se a gestão do conhecimento como o processo de obter, gerenciar e compartilhar a experiência e especialização dos colaboradores, com o objetivo de ter acesso a melhor informação no tempo certo. Estes conceitos baseiam que a "conversão do conhecimento" está relacionada à interação social, uma vez que é criado e expandido através das relações entre o conhecimento tácito e explícito ${ }^{5,25,28}$.

Aprofundando esta questão, observa-se que a gestão do conhecimento começa com o entendimento (saber-fazer) de um indivíduo, mas a criação de competência organizacional requer uma ligação e coordenação com os conhecimentos e capacidades de outros indivíduos ("articulação do conhecimento articulado"), e potencialmente de outras organizações. Para tanto a empresa deve atentar para: identificar a utilização estratégica do conhecimento; identificar o propósito do conhecimento; entender como transferir (difundir) o conhecimento; controlar a difusão, evitando que haja a perda da distinção competitiva (questão gerencial crítica) ${ }^{5,26-28}$.

Do ponto de vista estratégico, organizações hospitalares através de programas de gestão do conhecimento com o envolvimento de suas equipes multidisciplinares em parceria com as fontes pagadoras, são plenamente detentoras de vantagem competitiva. Entretanto, há de se observar o contexto organizacional que tende a capacitar ou criar barreiras à gestão do conhecimento. Assim, a cada dia, vem se utilizando da Medicina Baseada em Evidências (MBE), considerada como a integração das melhores evidências decorrentes de pesquisas cientificamente orientadas, com a habilidade clínica do médico responsável pela decisão e a preferência do paciente ${ }^{5,26-28}$.

Através da MBE, um método da organização de trabalho em saúde que visa vários resultados através da qualificação do processo decisório, pode-se utilizála como um critério de maior certeza de determinados achados e opiniões, 
apoiado em dados e informações cuja análise é feita dentro de padrões previamente estipulados ${ }^{26,27}$. O que sem a menor dúvida, organiza o processo do cuidado, identificando as diversas fases do processo assistencial, o que facilitará o processo de auditorias dos serviços prestados, e consequentemente, melhorar e ou até evitar glosas.

Observou-se nos estudos ( $n=11)$ que, principalmente os médicos, ao evoluírem e prescreverem os pacientes, determinam os procedimentos a serem executados. Entretanto, nem sempre os demais profissionais, inclusive outros médicos, entendem ou estão aptos a compreender a ordem desejada. Neste sentido, os protocolos assistenciais poderiam incentivar às equipes multidisciplinares a sistematizar a integração de todos, a partir de uma linguagem comum codificada e legítima, que, envolva também as fontes pagadoras no sentido de que entendam e apoiem o plano terapêutico, facilitando autorizações e, assim, evitando possíveis glosas.

Considerando que os protocolos médico-assistenciais são planos diagnósticos e terapêuticos para serem cumpridos, como instrumentos transparentes de avaliação, comunicação, orçamento, e, importantes ferramentas no processo da visualização da qualidade assistencial, servindo não só para antecipar os custos, assim como para evidenciar a eficácia dos resultados, nos estudos, observa-se que a construção e implementação dos protocolos médicoassistenciais, pode ser uma importante estratégia tática e operacional no ciclo do cuidado multiprofissional ${ }^{5,29,30}$.

Assim, os protocolos médico-assistenciais são formas "do que fazer e como fazer" em determinadas situações, são best practices que todos os hospitais/ instituições de saúde deveriam buscar para obter uma qualidade mandatória ${ }^{5,29,30}$.

Nos estudos selecionados, ainda não se observa a existência de referências do uso de protocolos assistenciais utilizados como elemento de qualidade nas auditorias das atividades multiprofissionais institucionais, e consequentemente, como uma ferramenta importante na melhoria de glosas. Parece ser ainda muito incipiente nas instituições/hospitais o conceito de processos assistenciais segundo evidências científicas como norteadores e sistematizadores do cuidado prestado, como uma cultura de resultado tanto na qualidade e segurança do paciente como na viabilização econômica advinda de um trabalho que deva seguir um fluxo e que seja adequadamente e corretamente registrado nos prontuários do paciente.

É importante, também mencionar que o foco de não conformidades relacionadas à falta de registros em prontuário, não devem ser focadas apenas nos serviços prestados ao paciente, mas, também, e principalmente, ao plano diagnóstico e terapêutico proposto pelas equipes multidisciplinares, que devem sem embasados em evidências científicas, que dão bases a sistematização de protocolos assistenciais ${ }^{5}$.

Observou-se que existe uma importante quantidade de artigos referentes a temática auditoria e a assistência prestada ao paciente, com foco nas não conformidades dos registros em prontuários e consequentes glosas. Mas, há 
ainda a necessidade de se aprofundar novos estudos que deem visibilidade a esse tema, especialmente por serem os registros assistenciais em prontuários importantes instrumentos de comunicação dos serviços prestados pela instituição de saúde/hospital, durante o internamento do paciente. Também se salientam os aspectos econômicos, legal, histórico, de ensino e pesquisa que são gerados, e que fornecem informações imprescindíveis para que a equipe multiprofissional possa agir em conjunto segundo objetivos comuns, que prioriza um cuidado eficiente, de qualidade, humanizado e com rastreabilidades.

Assim, é mister a necessidade de uma reflexão crítica da função-papel do enfermeiro e das equipes multidisciplinares, no âmbito hospitalar, acerca da auditoria não somente como uma ferramenta voltada aos interesses financeiros e políticos das instituições, mas, também, e, principalmente como uma estratégia na busca e conquista da qualidade do atendimento e assistência multiprofissional, focados em evidências científicas, utilizando a sistematização de protocolos assistenciais, que sem a menor dúvida, facilitarão o processo de coleta de informações da auditoria de prontuários.

Também é importante treinar equipes multidisciplinares para a melhoria dos registros em prontuários, não só na completude destes, mas, na sua legibilidade, processo este que depende de tempo e de mudanças, principalmente de hábitos por parte das diversas equipes, principalmente, os que registram em prontuários.

\section{CONSIDERAÇÕES FINAIS}

Com base na revisão realizada, pode-se observar que as instituições de saúde/hospitais estão evoluindo rapidamente em relação à qualidade dos serviços prestados, vislumbrando o baixo custo hospitalar através de equipes de profissionais qualificadas, com a visão financeira e holística do cliente, mas, que ainda não usam protocolos assistenciais baseados em evidências científicas como elementos de sistematizar a uniformização dos registros a serem auditados, evitando assim, as não conformidades nas informações obtidas, o que minimizariam as glosas hospitalares e a falta e/ou ilegibilidade de registros da assistência prestada relacionada à qualidade e segurança do paciente.

\section{DECLARAÇÃO DE PARTICIPAÇÃO}

Joana Angélica Santos Veloso Silva, Sylvia Lemos Hinrichsen, Kaline Assis Carneiro Brayner e Marcela Coelho Lemos contribuíram substancialmente para a concepção e planejamento do projeto, obtenção de dados ou análise e interpretação dos dados; 
Sylvia Lemos Hinrichsen e Tatiana de Aguiar Santos Vilella contribuíram significativamente na elaboração do rascunho ou na revisão crítica do conteúdo.

Sylvia Lemos Hinrichsen e Tatiana de Aguiar Santos Vilella participaram da aprovação da versão final do manuscrito.

\section{REFERÊNCIAS}

1. Goto DYN. Instrumento de Auditoria Técnica de Conta Hospitalar Mensurando Perdas e Avaliando a Qualidade da Assistência [monografia]. Curitiba: Universidade Federal do Paraná; 2001. Disponível em: http://bases.bireme.br/cgibin/wxislind.exe/iah/online/lsisScript=iah/iah.xis\&src=google\&base=LILACS\&lan $g=p \&$ nextAction=Ink\&exprSearch=315378\&indexSearch=ID Acesso em 01/09/16.

2. Paim CR, Ciconelli RM. Auditoria de Avaliação da Qualidade dos Serviços de Saúde. Rev Adm Saúde 2007; 9(36): 85-91.

3. Cortêz DT, Martins MR, Dela Torre SFS. Enfermagem em auditoria: uma revisão integrativa. Revista Saúde e Pesquisa 2013; 6(3):511-15.

4. Bacelar S; Almeida WM; Andrade GM. Falhas e dúvidas comuns no uso do prontuário do paciente. Brasília Médica 2002; 39(1/4): 42-51.

5. Hinrichsen SL. Qualidade \& Gestão de Riscos Segurança do Paciente. Rio de Janeiro: Medbook; 2012.

6. Galvão CF. Estudo do papel da auditoria de enfermagem para a redução dos desperdícios em materiais e medicamento. Mundo saúde (Impr.) 2002; 26(2): 275-82.

7. Riolino AN, Kliukas GBV. Relato de Experiência de Enfermeiras no Campo de Auditoria de Prontuário-uma ação inovadora. Revista Nursing 2003; 65(6): 35-38.

8.Rodrigues VA, Perroca MG, Jericó MC. Glosas hospitalares: importância das anotações de enfermagem. Arq Ciência Saúde 2004; 11(4): 210-14.

9. Luz A, Martins AP, Dynewicz AM. Características de anotações de enfermagem encontradas em auditoria. Rev Eletr Enf [Internet] 2007; 9(2): 34461.

10. Godoi NA, Machado CS, Lins MA, Cruz MG, Batista VM, Rosa BA. Auditoria de custo: análise comparativa das evidências de glosas em prontuário hospitalar. Rev Inst Ciênc Saúde 2008: 26(4): 403-8.

11. Scarparo AF, Ferraz CA. Auditoria em Enfermagem: Identificando sua concepção e métodos. Rev Bras Enferm 2008; 61(3): 302-5. 
12. Cianciarullo TI. Tendências. In: Cianciarullo TI. C \& Q: teoria e prática em auditoria de cuidados 1997; 10: 105-10.

13. Ferreira TS, Souza-Braga AL, Cavalcanti-Valente GS, Souza DF, CarvalhoAlves EM. Auditoria em enfermagem: o impacto das anotações de enfermagem no contexto das glosas hospitalares. Revista Aquichán 2009; 9(1): 38-49.

14. Mulatinho LM, Passos KFM, Torres LCGFL, Sousa PV. Auditoria de enfermagem: uma contribuição à minimização das glosas hospitalares. Revista de Pesquisa em Saúde 2012; 1(1): 35-40.

15. Franco MTG, Alcemi EM, Inocento MD. Avaliações dos registros de enfermeiro em prontuários de pacientes internados em unidade clínica. Acta paul enferm 2012; 25(2): 163-70.

16. Guerrer G, Lima AFC, Castilho V. Estudo da auditoria de contas em um hospital de ensino. Rev Bras Enferm 2015; 68(3): 358-63.

17. Brasil. Agência Nacional de Vigilância Sanitária. Manual de Acreditação das Organizações Prestadoras de Serviços Hospitalares. Brasília: 2000.

18. Santos MP, Rosa CDP. Auditoria de contas hospitalares: análise dos principais motivos de glosas em uma instituição privada. Revista da Faculdade de Ciências Médicas de Sorocaba. 2013; 15(4): 125-132.

19. Vituri DW; Matsuda LM. Os registros de enfermagem como indicadores da qualiade do cuidado: um estudo documental, descritivo-exploratório e retrospectivo. Online Braz J Nurs 2008; 7(1): 1-15.

20. Prado PR, Assis WALM. A importância das anotações de enfermagem nas glosas hospitalares. CuidArte Enferm 2011; 5(1): 62-68.

21. COFEN. Conselho Federal de Enfermagem. Resolução -COFEN No 266/2001. Dispõe sobre Atividades do Enfermeiro Auditor. Disponível em: http://www.cofen.gov.br/resoluo-cofen-2662001_4303.html Acesso em 18/10/16.

22. Scarparo AF. Auditoria em Enfermagem: revisão de literatura. Nursing 2005; 8(80): 46-50.

23. Dias TCL, Santos JLG, Cordenuzzi OCP, Prochnow AG. Auditoria em enfermagem: revisão sistemática da literatura. Rev Bras Enferm 2011; 64(5): 931-7.

24. Camelo SHH, Pinheiro A, Campos D, Oliveira TL. Auditoria de enfermagem e a qualidade da assistência à saúde: uma revisão da literatura. Rev. Eletr. Enf. [Internet] 2009; 11(4): 1018-25.

25. Falconi V. O Verdadeiro poder. práticas de gestão que conduzem a resultados revolucionários. Nova Lima: INDG Tecnologia e Serviços Ltda; 2009. 
26. Drummond JP et al. Medicina Baseada em Evidências: Novo Paradigma Assistencial e Pedagógico. 2 ed. São Paulo: Atheneu; 2002.

27. Castiel LD, Pávoa EC. Medicina Baseada em Evidências: "Novo paradigma assistencial e pedagógico?" Interface - Comunic Saúde Educ 2002; 6(11): 11732.

28. Silva SL. Gestão do conhecimento: uma revisão crítica orientada pela abordagem da criação do conhecimento. Ci. Inf 2004; 33(2): 143-151.

29. Makdissel M, Katz M, Corrêa AG, Forlenza LM, Perin MA, Júnior FSB, Nascimento TCD, Gomes IM, Franken M, Knobel M, Pesaro AEP, Santos OFP, Neto MC, Lottenberg CL. Efeito da implementação de um protocolo assistencial de infarto agudo do miocárdio sobre os indicadores de qualidade. Einstein. 2013;11(3): 357-63.

30. Brasil. Ministério da Saúde. Protocolos clínicos e diretrizes terapêuticas. Volume 2.Brasília 2010. Disponível em: http://bvsms.saude.gov.br/bvs/publicacoes/protocolos_clinicos_diretrizes_terap euticas_v2.pdf Acesso em 01/09/16.

Recebido: 06/01/2017; Aceito e publicado: 12/01/2017

Correspondência: Sylvia Lemos Hinrichsen - Rua Jornalista Guerra de Holanda, 158/2601 - Casa Forte - CEP 52061-010 - Recife (PE), Brasil - Email: sylviahinrichsen@hotmail.com

Fonte de financiamento: Não se aplica

Conflito de interesse: Não existe conflito de interesse

(C) This is an Open Access article distributed under the terms of the Creative Commons Attribution License, which permits unrestricted use, distribution, and reproduction in any medium, provided the original work is properly cited. 